\title{
Clinical efficacy of a systematic lung-protective protocol for COVID-19 pneumonia requiring invasive ventilation: A single-center retrospective study
}

Yoshihiko Takahashi ( $\square$ the.way.hope.goes.0907@gmail.com )

Maebashi Red Cross Hospital

Shu Utsumi

National Centre for Child Health and Development

Kenji Fujizuka

Maebashi Red Cross Hospital

Hiroyuki Suzuki

Maebashi Red Cross Hospital

Noritaka Ushio

Maebashi Red Cross Hospital

Yu Amemiya

Osaka Medical and Pharmaceutical University

Mitsunobu Nakamura

Maebashi Red Cross Hospital

\section{Research Article}

Keywords: COVID-19 pneumonia, intensive care unit, invasive ventilation, acute respiratory distress syndrome, lung-protective strategy, protocol

Posted Date: February 28th, 2022

DOI: https://doi.org/10.21203/rs.3.rs-1402410/v1

License: (a) (1) This work is licensed under a Creative Commons Attribution 4.0 International License. Read Full License 


\section{Abstract \\ Background}

The benefits of introducing a systematic lung-protective protocol for patients with coronavirus disease (COVID-19) pneumonia and who require invasive ventilation in the intensive care unit (ICU) are unknown. Herein, we aimed to evaluate the clinical effects of introducing such a protocol in the context of mortality, duration of ventilation, and length of ICU stay.

\section{Methods}

In this single-center, retrospective, quality comparison study, we identified patients with COVID-19 pneumonia who received invasive ventilation in our ICU between February 2020 and October 2021. We established a systematic lung-protective protocol for the pre-introduction group until March 2021 and the post-introduction group after April 2021. Patients who did not receive invasive ventilation and who underwent veno-venous extracorporeal membrane oxygenation in a referring hospital were excluded. We collected patient characteristics at the time of ICU admission, including age, sex, body mass index (BMI), comorbidities, sequential organ failure assessment (SOFA) score, acute physiology and chronic health evaluation II (APACHE II) score, and Murray score. The study outcomes were ICU mortality, length of ICU stay, and duration of ventilation.

\section{Results}

The pre-introduction and post-introduction groups included 18 and 50 patients, respectively. No significant differences were observed in sex, BMI, SOFA score, APACHE II score, and Murray score; however, age was lower in the post-introduction group (70 vs. $56, p=0.003)$. The introduction of this protocol did not improve ICU mortality. However, it reduced the ICU length of stay ( 26 days vs. 11 days, $p$ $=0.003$ ) and tended to shorten the duration of ventilation ( 15 days vs. 10 days, $p=0.06$ ).

\section{Conclusions}

The introduction of the protocol was associated with a decrease in the length of ICU stay and duration of ventilation; however, it did not change mortality. The application of the protocol could improve the security of medical resources during the COVID-19 pandemic. Further prospective multicentre studies are needed.

\section{Background}

The coronavirus disease (COVID-19) pandemic has spread at an overwhelming rate and has caused many deaths [1]. The severity of COVID-19 pneumonia varies, and most cases are mild and non-fatal [2]. 
However, the most severe cases requiring invasive ventilation, which will be addressed as severe COVID19 pneumonia, meet the criteria for acute respiratory distress syndrome (ARDS) and have a high fatality rate [3]. Since these patients require intensive care, each country needs to secure intensive care unit (ICU) resources and provide effective treatments [4].

Severe COVID-19 pneumonia has been found to share many similarities with ARDS in terms of respiratory mechanics [5]. Therefore, lung-protective strategies, such as lung-protective ventilation with continuous intravenous infusion of a neuromuscular blocking agent, early application of prone positioning, and prolonged prone positioning, could be effective not only for patients with ARDS but also for those with severe COVID-19 pneumonia [6-10].

It would be valuable to establish a systematic protocol for severe COVID-19 pneumonia and therefore we implemented a lung-protective strategy. The benefits of introducing a protocol for severe COVID-19 pneumonia remain unknown. The aim of our study was to evaluate the clinical effects of a systematic lung-protective protocol for COVID-19 pneumonia in the context of mortality, duration of ventilation, and length of ICU stay.

\section{Methods}

\section{Study setting}

This was a single-center, retrospective, quality comparison study with a systematic lung-protective protocol conducted at Maebashi Red Cross Hospital. The study period was from February 2020 to October 2021, with the pre-introduction group until March 2021, and the post-introduction group after April 2021. We identified patients over 18-years-old with COVID-19 pneumonia in our ICU. Patients who did not receive invasive ventilation, had contraindications for prone positioning (see Additional File 1), and those who underwent veno-venous extracorporeal membrane oxygenation (V-V ECMO) in a referring hospital were excluded. Our study was approved by the Ethics Committee of the Maebashi Red Cross Hospital (ID: 2021-51), which waived the requirement for informed consent from patients and their relatives, given the retrospective and observational nature of the study.

\section{Data collection}

Data was collected as follows: $(\nabla)$ patient characteristics at the time of ICU admission included the following: age, sex, body mass index, comorbidities, sequential organ failure assessment (SOFA) score, acute physiology and chronic health evaluation II (APACHE II) score, Murray score [11]; (区) medications received during ICU stay; ( () availability of treatment with V-V ECMO; ( () ventilator parameters within two days after ICU admission (tidal ventilation volume/predicted body weight, driving pressure, plateau airway pressure, positive end-expiratory pressure), induction time and average duration time of prone positioning, and presence of complications associated with prone positioning; ( () the incidence of ventilator-associated pneumonia (VAP) as defined in previous literature [12]. These data were collected retrospectively from electronic medical records. 


\section{Outcome measures}

The primary outcome was mortality in the ICU. The secondary outcomes were duration of ventilation and length of stay in the ICU. Ventilation duration was defined as the absence of reintubation or the use of noninvasive ventilation within $48 \mathrm{~h}$ of extubation. For patients with tracheostomy, we defined weaning from the ventilator as the ability to breathe without assistance from a tracheostomy cannula for more than $24 \mathrm{~h}[6]$.

\section{Protocol}

The eligible patients were included in the protocol immediately after ICU admission.

Firstly, after ICU admission, all patients were introduced to a high-flow nasal cannula, and oxygenation, respiratory acidosis, and respiratory effort were assessed. The criteria for intubation were as follows: Patients met one of the conditions $\mathrm{PaO}_{2} / \mathrm{F}_{1} \mathrm{O}_{2}<150 \mathrm{mmHg}$ [13], respiratory acidosis with $\mathrm{pH}<7.30$, or excessive respiratory efforts. Secondly, patients were placed in prone positioning with lung-protective ventilation and administered continuous intravenous infusion of rocuronium, a neuromuscular blocking agent, promptly after intubation.

The target parameters of lung-protective ventilation were as follows: (i) tidal ventilation volume at 6 $\mathrm{mL} /$ predicted body weight [14], (ii) driving pressure $<15 \mathrm{~cm} \mathrm{H}_{2} \mathrm{O}$ [15], and (iii) plateau airway pressure < $30 \mathrm{~cm} \mathrm{H}_{2} \mathrm{O}$ [16]. In addition, the positive end-expiratory pressure (PEEP) was determined according to the lower PEEP/higher $\mathrm{F}_{1} \mathrm{O}_{2}$ table proposed by the ARDS Clinical Trials Network [17]. The targets of prone positioning were as follows: (i) early prone positioning: the application of prone positioning within $36 \mathrm{~h}$ after intubation, and (ii) prolonged prone positioning: average duration being at least $16 \mathrm{~h}$ per session [6]. Thirdly, prone positioning was discontinued when $\mathrm{PaO}_{2} / \mathrm{F}_{1} \mathrm{O}_{2}>200 \mathrm{mmHg}$ could be maintained for more than $4 \mathrm{~h}$ in the supine position. It was discontinued using a neuromuscular blocking agent simultaneously when prone positioning was completed.

Even if $\mathrm{PaO}_{2} / \mathrm{F}_{1} \mathrm{O}_{2}>200 \mathrm{mmHg}$ could not be achieved, the continuation of prone positioning was limited to $96 \mathrm{~h}$ after intubation, at which point the need for V-V ECMO was discussed [18].

Fourthly, patients were slowly weaned off the respiratory setting by adjusting the respiratory setting and sedatives and if necessary, using a neuromuscular blocking agent for respiratory effort and ventilation asynchrony.

Finally, the protocol was withdrawn if they met the criteria for the initiation of spontaneous awakening test and spontaneous breathing test [19]. In addition, the protocol was terminated when V-V ECMO was introduced (Fig. 1). Drug therapy during the protocol is shown separately in Additional File 2.

\section{Statistical analysis}


Distributed continuous variables without a normal distribution were presented as median and interquartile range. Categorical data were summarized as numbers or percentages. For univariate analysis, the Mann-Whitney $U$ test for continuous variables and Fisher's exact test for categorical variables were used for comparison. For multivariate analysis, if the residues did not follow a normal distribution, they were log-transformed and subjected to multiple regression analysis, adjusting for significant covariates at baseline. Data were assumed to be missing at random, with no imputation or interpolation of the missing values. Statistical tests were two-tailed, and statistical significance was set at $p<0.05$. All statistical analyses were performed using EZR [20], a graphical user interface for $R(R$ Foundation for Statistical Computing, Vienna, Austria).

\section{Results}

\section{Baseline characteristics}

A total of 96 patients were admitted to our ICU, and 68 patients who received invasive

ventilation were eligible for our study (Figure 2). Eighteen patients were included in the pre-introduction group, and 50 patients were included in the post-introduction group. Table 1 summarizes the patients' characteristics. There were no significant differences between the groups in sex, body mass index, SOFA score, APACHE II score, or Murray score; however, age was lower in the post-introduction group (70 vs. 56, $P=0.003)$. The type of antiviral drug used changed over time in the two groups, while the use of corticosteroids was not different. The incidence of VAP was not different between the two groups (OR, $0.64 ; 95 \% \mathrm{Cl}, 0.18 \square 2.16)$, although the use of antibiotics was higher in the post-introduction group $(p<0.001)$. The rate of $\mathrm{V}-\mathrm{V}$ ECMO significantly reduced from $33 \%$ to $2 \%$ in the post-introduction group (OR, $0.04 ; 95 \% \mathrm{Cl}, 0.0100 .41)$.

\section{Lung-protective strategy}

Table 2 shows the parameters of ventilation, prone positioning, and complications associated with prone positioning. The parameters of lung-protective ventilation did not differ between the two groups. The achievement of early prone positioning increased from $56 \%$ to $98 \%$ in the post-introduction group, and the average duration of prone positioning significantly increased from $15 \mathrm{~h}$ to $18 \mathrm{~h}$ in the post-introduction group $(p=0.007)$. Although the duration of prone positioning increased, there was no increase in the number of complications in the post-introduction group.

\section{Primary and Secondary outcomes}

The results of outcomes are shown in Table 3. The mortality rate in the ICU showed a decreasing trend from $17 \%$ to $4 \%$; however, it did not show statistical significance (OR, $0.21 ; 95 \% \mathrm{Cl}, 0.0102 .05)$. The duration of ventilation was reduced from 15 days to 10 days, with statistical significance in the univariate analysis; however, it did not remain statistically significant after adjusting for age, sex, and SOFA score (see Additional File 3). The length of stay in the ICU was reduced from 33 to 11 days, with statistical 
significance. Even after adjusting for age, sex, and SOFA score, the differences remained statistically significant ( $p=0.003$, see Additional File 4).

\section{Discussion}

\section{Overview}

The introduction of the protocol for COVID-19 pneumonia requiring invasive ventilation did not improve mortality in the ICU; however, it reduced the length of ICU stay and shortened the duration of ventilation.

\section{Mortality}

In our study, mortality in the ICU did not improve by introducing the protocol. This may be because the rate of V-V ECMO tended to be higher in the pre-introduction group. The results of V-V ECMO treatments for COVID-19 pneumonia have been good, according to a report by the Extracorporeal Life Support Organization [21]. The high rate of V-V ECMO may have positively affected mortality in the preintroduction group. However, it is important to note that despite the lack of difference in severity between the two groups, the rate of V-V ECMO was reduced by introducing the protocol, and ICU mortality was not worse in the post-introduction group. In addition, ICU mortality tended to be lower in our hospital than reported in previous studies. For example, the overall mortality of $7 \%$ in the ICU in our cohort was lower compared with 38\% reported in the PRoVENT-COVID cohort (Table 3) [22]. The small number of death events may have contributed to the lack of statistically significant differences. Therefore, it is important that the introduction of the protocol did not worsen mortality with no increase in V-V ECMO initiation.

\section{Length of ICU stay and duration of ventilation}

The protocol reduced the length of ICU stay and ventilation duration. In our study, despite no difference in the rate of achievement of lung-protective ventilation in the two groups, the achievement of early applied prone positioning and the duration of prone positioning increased significantly, suggesting that these approaches could be effective. In the PROSEVA study, the achievement of early prone positioning and the duration of prone positioning reduced the length of stay in the ICU and duration of ventilation [6]. Therefore, the results of our study are consistent with those of the PROSEVA study. Therefore, the introduction of the protocol could comply with these approaches, contributing to an improvement in the length of stay in the ICU and the duration of ventilation.

\section{Adverse events associated with prone positioning}

There were no significant differences between the groups with respect to adverse events associated with prone positioning in our study, as well as in the PROSEVA study [6]. The low incidence of adverse events could be related to the fact that our hospital and the facilities that participated in PROSEVA study were familiar with the management of severe respiratory failure.

\section{Limitation}


In addition to our study design being single-centered and retrospective, the mutation, prevalence of COVID-19, and drug treatment strategy for COVID-19 pneumonia changed significantly before and after the introduction of the protocol. This could have influenced the results, and the number of cases was not sufficient to adequately adjust for the confounding factors. Despite these limitations, during a pandemic, this protocol could contribute to securing medical resources by reducing the length of ICU stay and duration of ventilation. With no increasing adverse events, this protocol could be generalised since it does not require special drugs or equipment but only prone positioning.

\section{Conclusions}

The introduction of the protocol for COVID-19 pneumonia patients requiring invasive ventilation was associated with improved length of stay in the ICU and duration of ventilation; however, the difference was not statistically significant. Nevertheless, the protocol could contribute to the efficient use of medical resources during the pandemic. Further investigation regarding the efficacy of the protocol using a multicentre prospective study is required.

\section{Declarations}

- Ethics approval and consent to participate

Our study was approved by the ethics committee of the Maebashi Red Cross Hospital (ID: 2021-51), which waived the requirement for informed consent from patients and their relatives, given the retrospective and observational nature of the study.

- Consent for publication

We made an opt-out regarding our study on the website of Maebashi Red Cross Hospital. In the case of minors, adults who are objectively judged to lack sufficient judgment, adults who are unconscious or in an emergency and life-threatening situation, adults who require consideration of the name of the disease, and others (including the deceased), a substitute will be requested. The substitute should be a person who has parental authority over the subject, a spouse, a guardian, or other similar person.

- Availability of data and materials

The dataset supporting the conclusions of this article is available upon reasonable request from the authors.

- Competing interests

The authors declare that they have no competing interests.

- Funding 
This study was conducted with no specific support.

- Authors' contributions

Yoshihiko Takahashi: Conceptualization, Methodology, Resources, Investigation, Formal analysis, Writing

Shu Utsumi: Conceptualization, Methodology, Investigation, Writing

Kenji Fujizuka: Conceptualization , Resources

Hiroyuki Suzuki: Conceptualization, Resources

Noritaka Ushio: Conceptualization , Resources

Yu Amemiya: Conceptualization, Resources

Mitsunobu Nakamura: Supervision, Project administration

All authors read and approved the final manuscript.

- Acknowledgements

We thank the doctors, nurses, nutritionists, pharmacists, and physical therapists in the ICU of Maebashi Red Cross Hospital.

\section{References}

1. Berlin DA, Gulick RM, Martinez FJ. Severe Covid-19. N Engl J Med. 2020;383:2451-60. https://doi.org/10.1056/NEJMcp2009575

2. Wu Z, McGoogan JM. Characteristics of and important lessons from the coronavirus disease 2019 (COVID-19) outbreak in China: summary of a report of 72314 cases from the Chinese Center for Disease Control and Prevention. JAMA. 2020;323:1239-42. https://doi.org/10.1001/jama.2020.2648

3. Gibson PG, Qin L, Puah SH. COVID-19 acute respiratory distress syndrome (ARDS): clinical features and differences from typical pre-COVID-19 ARDS. Med J Aust. 2020;213:54-56.e1. https://doi.org/10.5694/mja2.50674

4. Litton E, Bucci T, Chavan S, Ho YY, Holley A, Howard G, et al. Surge capacity of intensive care units in case of acute increase in demand caused by COVID-19 in Australia. Med J Aust. 2020;212:463-7. https://doi.org/10.5694/mja2.50596

5. Fan E, Beitler JR, Brochard L, Calfee CS, Ferguson ND, Slutsky AS, et al. COVID-19-associated acute respiratory distress syndrome: is a different approach to management warranted? Lancet Respir Med. 2020;8:816-21. https://doi.org/10.1016/S2213-2600(20)30304-0 
6. Guérin C, Reignier J, Richard JC, Beuret P, Gacouin A, Boulain T, et al. Prone positioning in severe acute respiratory distress syndrome. N Engl J Med. 2013;368:2159-68.

https://doi.org/10.1056/NEJMoa1214103

7. Papazian L, Forel JM, Gacouin A, Penot-Ragon C, Perrin G, Loundou A, et al. Neuromuscular blockers in early acute respiratory distress syndrome. N Engl J Med. 2010;363:1107-16. https://doi.org/10.1056/NEJMoa1005372

8. Shelhamer MC, Wesson PD, Solari IL, Jensen DL, Steele WA, Dimitrov VG, et al. Prone positioning in moderate to severe acute respiratory distress syndrome due to COVID-19: A cohort study and analysis of physiology. J Intensive Care Med. 2021;36:241-52.

https://doi.org/10.1177/0885066620980399

9. Mathews KS, Soh H, Shaefi S, Wang W, Bose S, Coca S, et al. Prone positioning and survival in mechanically ventilated patients with coronavirus Disease 2019-related respiratory failure. Crit Care Med. 2021;49:1026-37. https://doi.org/10.1097/CCM.0000000000004938

10. Alhazzani W, Møller MH, Arabi YM, Loeb M, Gong MN, Fan E, et al. Surviving sepsis campaign: guidelines on the management of critically ill adults with coronavirus disease 2019 (COVID-19). Intensive Care Med. 2020;46:854-87. https://doi.org/10.1007/s00134-020-06022-5

11. D'Negri CE, de Vito EL. Making it possible to measure knowledge, experience and intuition in diagnosing lung injury severity: a fuzzy logic vision based on the Murray score. BMC Med Inform Decis Mak. 2010;10:70. https://doi.org/10.1186/1472-6947-10-70

12. Rouzé A, Martin-Loeches I, Povoa P, Makris D, Artigas A, Bouchereau M, et al. Relationship between SARS-CoV-2 infection and the incidence of ventilator-associated lower respiratory tract infections: a European multicenter cohort study. Intensive Care Med. 2021;47:188-98. https://doi.org/10.1007/s00134-020-06323-9

13. Bellani G, Laffey JG, Pham T, Madotto F, Fan E, Brochard L, et al. Noninvasive ventilation of patients with acute respiratory distress syndrome. Insights from the LUNG SAFE study. Am J Respir Crit Care Med. 2017;195:67-77. https://doi.org/10.1164/rccm.201606-13060C

14. Acute Respiratory Distress Syndrome Network, Brower RG, Matthay MA, Morris A, Schoenfeld D, Thompson BT, et al. Ventilation with lower tidal volumes as compared with traditional tidal volumes for acute lung injury and the acute respiratory distress syndrome. N Engl J Med. 2000;342:1301-8. https://doi.org/10.1056/NEJM200005043421801

15. Amato MBP, Meade MO, Slutsky AS, Brochard L, Costa ELV, Schoenfeld DA, et al. Driving pressure and survival in the acute respiratory distress syndrome. N Engl J Med. 2015;372:747-55. https://doi.org/10.1056/NEJMsa1410639

16. Marini JJ, Gattinoni L. Management of COVID-19 respiratory distress. JAMA. 2020;323:2329-30. https://doi.org/10.1001/jama.2020.6825

17. Brower RG, Lanken PN, Maclntyre N, Matthay MA, Morris A, Ancukiewicz M, et al. Higher versus lower positive end-expiratory pressures in patients with the acute respiratory distress syndrome. $\mathrm{N} \mathrm{Engl} \mathrm{J}$ Med. 2004;351:327-36. https://doi.org/10.1056/NEJMoa032193 
18. Bartlett RH, Ogino MT, Brodie D, McMullan DM, Lorusso R, Maclaren G, et al. Initial ELSO guidance document: ECMO for COVID-19 patients with severe cardiopulmonary failure. ASAIO J. 2020;66:4724. https://doi.org/10.1097/MAT.0000000000001173

19. Girard TD, Kress JP, Fuchs BD, Thomason JW, Schweickert WD, Pun BT, et al. Efficacy and safety of a paired sedation and ventilator weaning protocol for mechanically ventilated patients in intensive care (Awakening and Breathing Controlled trial): a randomised controlled trial. Lancet. 2008;371:126-34. https://doi.org/10.1016/S0140-6736(08)60105-1

20. Kanda Y. Investigation of the freely available easy-to-use software 'EZR' for medical statistics. Bone Marrow Transplant. 2013;48:452-8. https://doi.org/10.1038/bmt.2012.244

21. Barbaro RP, MacLaren G, Boonstra PS, Iwashyna TJ, Slutsky AS, Fan E, et al. Extracorporeal membrane oxygenation support in COVID-19: an international cohort study of the Extracorporeal Life Support Organization registry. Lancet. 2020;396:1071-8. https://doi.org/10.1016/S01406736(20)32008-0

22. Botta M, Tsonas AM, Pillay J, Boers LS, Algera AG, Bos LDJ, et al. Ventilation management and clinical outcomes in invasively ventilated patients with COVID-19 (PRoVENT-COVID): a national, multicentre, observational cohort study. Lancet Respir Med. 2021;9:139-48. https://doi.org/10.1016/S2213-2600(20)30459-8

23. WHO Rapid Evidence Appraisal for COVID-19 Therapies (REACT) Working Group, Sterne JAC, Murthy S, Diaz JV, Slutsky AS, Villar J, et al. Association between administration of systemic corticosteroids and mortality among critically ill patients with COVID-19: A meta-analysis. JAMA. 2020;324:133041. https://doi.org/10.1001/jama.2020.17023

24. Beigel JH, Tomashek KM, Dodd LE, Mehta AK, Zingman BS, Kalil AC, et al. Remdesivir for the treatment of Covid-19 - final report. N Engl J Med. 2020;383:1813-26. https://doi.org/10.1056/NEJMoa2007764

25. REMAP-CAP Investigators; ACTIV-4a Investigators; ATTACC Investigators; Ewan CG, Charlotte AB, Bryan JM, Patrick RL, Jeffrey SB, Michelle NG, et al. Therapeutic Anticoagulation with Heparin in Critically III Patients with Covid-19. N Engl J Med. 2021;385:777-89. https://doi.org/10.1056/NEJMoa2103417

\section{Abbreviations}

COVID-19, coronavirus disease 2019; ICU, intensive care unit; ARDS, acute respiratory distress syndrome; V-V ECMO, veno-venous extracorporeal membrane oxygenation, BMI, body mass index; SOFA, sequential organ failure assessment; APACHE II, Acute Physiology and Chronic Health Evaluation II; VAP, ventilatorassociated pneumonia; PEEP, positive end-expiratory pressure

\section{Tables}

Tables 1-3 are in the supplementary files section. 


\section{MAEBASHI COVID-19 PROTOCOL}

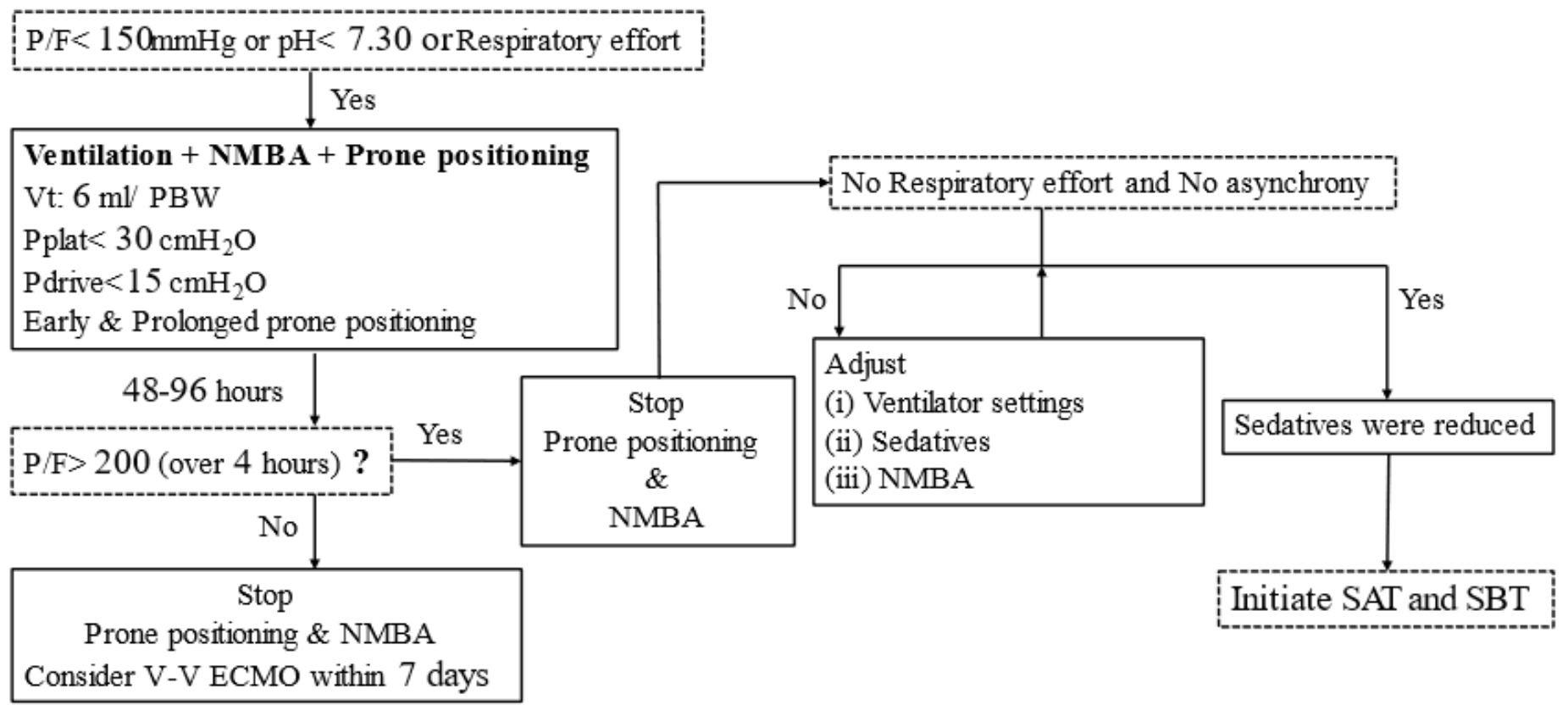

Figure 1

Maebashi COVID-19 Protocol.

Definition of abbreviations: $\mathrm{P} / \mathrm{F}=\mathrm{PaO} 2 / \mathrm{FIO2}$; $\mathrm{PBW}=$ predicted body weight; $\mathrm{Vt}=$ Tidal Volume $(\mathrm{mL})$; Pplat=plateau airway pressure; NMBA=neuromuscular blocking agent; $\mathrm{V}-\mathrm{V} E \mathrm{EMO}=$ veno-venous extracorporeal membrane oxygenation; $\mathrm{SAT}=$ spontaneous awakening test; $\mathrm{SBT}=$ spontaneous breathing test

Maebashi COVID-19 Protocol (included the lung-protective strategy): Lung-protective ventilation with neuromuscular blocking agents, early application of prone positioning, and prolonged prone positioning. 


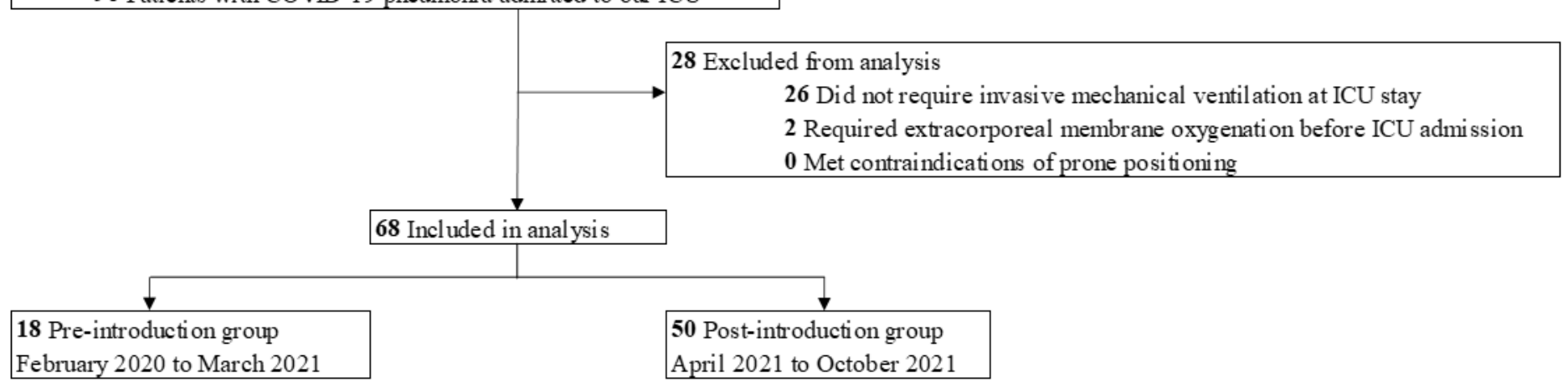

\section{Figure 2}

Study flow.

We identified 96 patients over 18-years-old with coronavirus disease 2019 (COVID-19) pneumonia in our intensive care unit; 26 patients did not receive invasive ventilation, and 2 patients who underwent venovenous extracorporeal membrane oxygenation in a referring hospital were excluded. A total of 68 patients were included in the analysis, with 18 in the pre-introduction group and 50 in the post-introduction group.

\section{Supplementary Files}

This is a list of supplementary files associated with this preprint. Click to download.

- Table1.pptx

- Table2.pptx

- Table3.pptx

- Additionalfile1.pptx

- Additionalfile2.pptx

- Additionalfile3.pptx

- Additionalfile4.pptx

- AdditionalFiles.docx 